\title{
Prognostic Features and Treatment of Endocervical Adenocarcinoma of the Cervix ${ }^{1}$
}

\author{
Michael P. Hopkins, M.D., Patrick Sutton, M.D., \\ AND James A. RoBerts, M.D. \\ Department of Obstetrics and Gynecology University of Michigan, Ann Arbor, Michigan 48109
}

Received January 27, 1986

\begin{abstract}
Adenocarcinoma of the cervix represents an increasing percentage of cervical cancers as well as a controversial clinical problem. One hundred seventy-two cases of adenocarcinoma were treated at The University of Michigan Medical Center from 1970 to 1984, representing $16.8 \%$ of cervical cancers. Of this group 84 patients were diagnosed with endocervical columnar cell type and form the study population for this report. Survival was influenced by stage, grade, and nodal metastases. Age did not influence survival. Survival in stage I disease was not influenced by treatment modality. Radical surgery or radiation therapy provided equal survival and ovarian conservation did not adversely influence survival. Radical surgery can be considered a primary means of therapy in young women and offers the same advantages over radiation therapy as those offered for squamous cell disease. (6) 1987 Academic Press, Inc
\end{abstract}

\section{INTRODUCTION}

Adenocarcinoma of the cervix appears to be increasing from its historic 5\% of all cervical cancers $[1,2]$. Historically it has portended a poorer prognosis in all stages than its squamous counterpart [1]. The treatment of this pathologic type of cancer is controversial and prognostic features are still being defined. This particular entity has been recognized at The University of Michigan for over 50 years. A review of the clinical characteristics and treatment of endocervical adenocarcinoma at The University of Michigan Medical Center was undertaken for the time period 1970 to 1984 to delineate prognostic features and therapeutic alternatives.

\section{MATERIAL AND METHODS}

The clinical records, pathology reports, and Gynecologic Tumor Conference notes for all patients registered at The University of Michigan Medical Center Tumor Registry with the diagnosis of adenocarcinoma of the cervix for the time period 1970 to 1984 were reviewed. Information was recorded on clinical presentation, medical history, height, weight, oral contraceptive usage, treatment modality, follow-up, grade, and size of cervical lesion. All of the pathologic material during this time period had been presented at The Gynecologic Tumor

' Presented at the Western Association of Gynecologic Oncologists (WAGO), June 1985. 


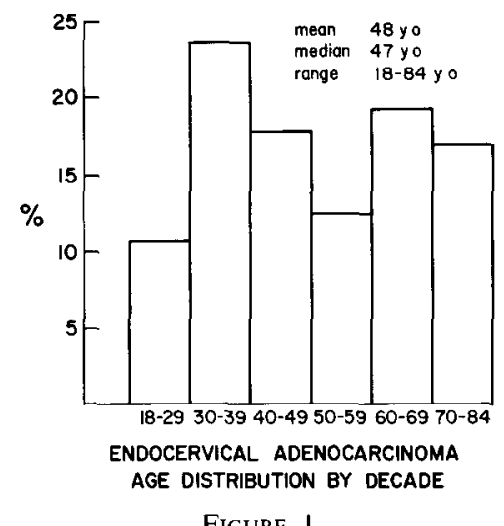

Figure I

Conference and reviewed by one of threc gynecologic pathologists. Cascs where pathology was reviewed in consultation only were not included in this study. All cases were staged according to International Federation of Gynecologists and Obstetricians (FIGO) guidelines. Cases were excluded where the primary site could not be differentiated between an endocervical and an endometrial origin.

Statistical analysis was performed by the Department of Biostatistics, School of Public Health, University of Michigan. Survival was plotted with the life table method described by Kaplan and Meier [3]. The Wilcoxan test was used to test difference in survival [4]. A Cox model multivariant regression analysis was used to compare hazard for tumor-related death related to potential risk factors [5]. A normal approximation ( $Z$ score) was used to test the significance of the clinical characteristics.

\section{RESULTS}

During this 14-year time period 172 new cases of invasive cervical adenocarcinoma were evaluated and treated at The University of Michigan Medical Center. The total number of new cases of cervical cancer treated during this time period was 1072; thus the adenocarcinomas represented $16.8 \%$ of the total. Eighty-four of these were of the endocervical columnar cell type and form the study population for this paper. The remaining 88 were composed of adenosquamous, papillary, clear cell, or mucoid type pathology.

The age distribution was relatively uniform throughout the decades with the highest percentage of cases diagnosed in their thirties (Fig. 1). The clinical characteristics of the population are presented in Table 1. Patients were classified as obese when their weight was $20 \%$ above the ideal weight for large frame females. Oral contraceptive usage was recorded only for those under 45 years old who were using oral contraceptives continuously for 1 or more years at the time of diagnosis.

The majority of the study population (55\%) had been examined by a physician within 1 year of diagnosis and $68 \%$ had been examined within 3 years. The most 
TABLE 1

Clinical Characteristics of Study Population

\begin{tabular}{lr}
\hline & $\%$ \\
\hline Married & 94 \\
Multiparous & 80 \\
Obese & 20 \\
Iypertensive & 27 \\
Diabetic & 8 \\
Oral contractive user & \\
$\quad<45$ years old & 47 \\
\hline
\end{tabular}

common presenting symptom (71\%) was abnormal bleeding. In those patients having cytology, only $60 \%$ showed an abnormality.

Survival analysis was performed for all stages of disease evaluating the effect of stage and grade. The overall 5-year survival was $52 \%$. Survival was $58 \%$ for stages I, II at 7 years. Those patients with advanced disease stages III, IV did very poorly with $58 \%$ dying within 1 year of diagnosis $(P=.0001)$ (Fig. 2). Twenty-eight patients with well-differentiated lesions enjoyed a significantly better survival advantage over those with a moderate or poorly differentiated tumor $(P=.0002)$ (Fig. 3).

An in situ component, either adeno or squamous in origin, was present in $20 \%$ of the cases. The presence of this in situ component as an independent finding influenced prognosis. Seventeen patients with an in situ component had a $90 \%$ 5 -year survival while 67 patients without this component had a 5-year survival of approximately $40 \%$.

A separate survival analysis was then performed on the 68 patients with stage I and II disease evaluating for age, node status, size, and grade. Size and node

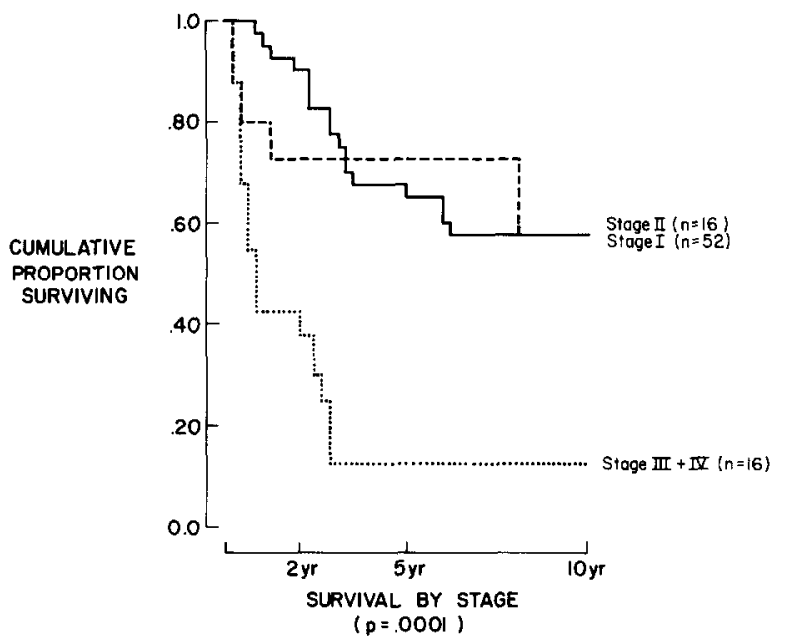

Figure 2 


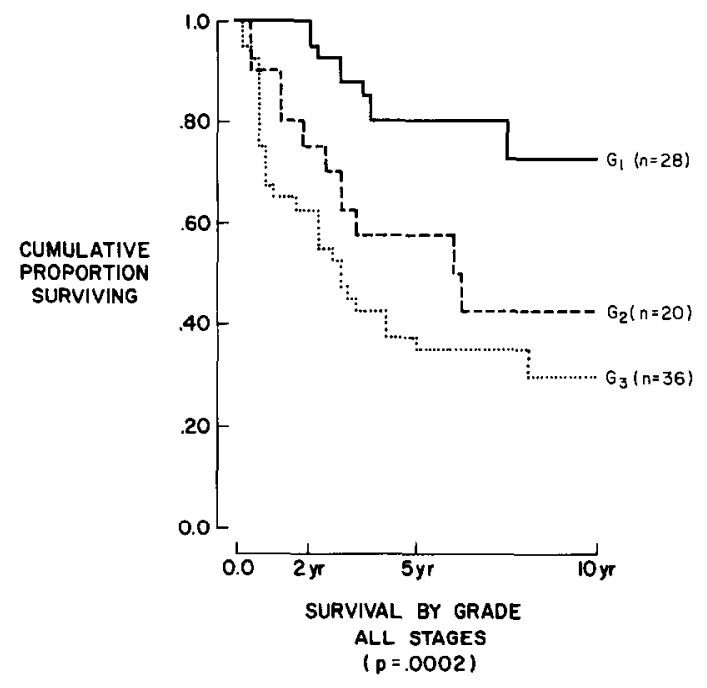

Figure 3

status evaluated independently were both found to significantly influence survival. Node status was available in 43 patients and was found to significantly influence survival. Thirty-four patients with negative nodes enjoyed an $88 \% 5$-year survival while all those with positive nodes were dead of disease by 5 years except one who was dead by 7 years. Forty-one patients with lesions less than $3 \mathrm{~cm}$ in size enjoyed a significant survival advantage $(P=0.0022)$ over those with lesions greater than $3 \mathrm{~cm}$ in size. However, when a Cox model multivariant analysis evaluating these factors was performed, metastatic disease to the lymph nodes was found to be the only significant prognostic factor (Table 2).

In stage I disease a comparison of treatment modalities was undertaken. The cumulative 5-year survival was equal for those treated by radiation therapy, radical surgery, or combined radiation therapy with extrafascial hysterectomy. The poorest survival was in the subgroup of patients treated with radical hysterectomy who then received adjuvant external radiation therapy for factors such as metastatic nodal disease, angiolymphatic invasion, or involved surgical margins. Eight patients had their ovaries conserved and this did not adversely effect their survival compared to the sixteen patients who had their ovaries removed (Table $3)$.

TABLE 2

Age, Tumor Size, and Node Status Related to Survival Stages I and II

\begin{tabular}{lc}
\hline Characteristic & $P$ value \\
\hline Age & 0.7621 \\
Size $>3 \mathrm{~cm}$ & 0.1226 \\
Node metastases & 0.0016 \\
\hline
\end{tabular}


TABLE 3

Stage I Survival and Type of Therapy Employed

\begin{tabular}{lcc}
\hline Rx & No. & $\begin{array}{c}\text { 5-year survival } \\
(\%)\end{array}$ \\
\hline Radical hysterectomy & 8 & 100 \\
Radical hysterectomy + BSO & 16 & 67 \\
Radical hysterectomy + post-op RT & 6 & 16 \\
Radiation therapy with extrafacial hysterectomy & 7 & 50 \\
Radiation therapy & 15 & 62 \\
\hline
\end{tabular}

\section{DISCUSSION}

Adenocarcinoma is apparently increasing and there is some question whether this is an absolute or relative increase secondary to the decrease in invasive squamous cell carcinoma. At The University of Michigan Medical Center (UMMC) previous reports have shown a 5.5 and $9.6 \%$ incidence of adenocarcinoma of the cervix $[1,6]$. This report shows a further increase with this neoplasm now representing one out of six cases of cervical cancer treated at UMMC. The mean number of cases per year has also increased by twofold [Table 4].

Various subtypes of adenocarcinoma have been reported to influence prognosis with the endocervical type being the most favorable [7]. A recent report, however, failed to find a difference among subtypes [8]. This study includes only those patients with endocervical columnar cell type adenocarcinoma. The 5-year survival of $52 \%$ for all stages is very similar to the $51 \%$ reported for this type from the Norwegian Radium Hospital [9]. It is also consistent with the previous 5-year survival reported from this institution [1]. The 5-year survival in stage I disease of $68 \%$ is similar to previous reports from the UMMC of $71 \%$. The survival in stage II disease, however, has increased considerably with the use of radiation therapy alone and represents an improvement in survival to $58 \%$ from 33 and $25 \%$ previously reported from the UMMC $[1,6]$. Unfortunately advanced stages III and IV still represent a very poor prognosis with conventional radiation therapy offering little hope for salvage. The $9 \%$ survival in stage III disease is remarkably similar to the $15 \%$ survival reported by Abell. No patient with stage IV disease in either series has survived [6].

A large percentage of the patients were married and had children. Obesity and hypertension were not increased over estimates of the national population where obesity is $20 \%$ and hypertension $26 \%$. The incidence of diabetes is estimated at

TABLE 4

Mean Number of New Cases and Percentage of Newly Diagnosed Cervical Adenocarcinomas at UMMC by Studid Period

\begin{tabular}{lccc}
\hline Author & Years & Cases/year & \% Total \\
\hline Abell + Gosling & $1931-1954$ & 5.52 & 5.5 \\
Gallup + Abell & $1965-1970$ & 4.6 & 9.6 \\
Present & $1970-1984$ & 12.2 & 16.8 \\
\hline
\end{tabular}


$4 \%$ in the national population, and the $8 \%$ in the present study population is not significantly increased $(P=0.37)[10]$. Oral contraceptive usage has been noted with adenocarcinoma [1]. No study has been done to conclusively show a causal relationship and a large case control study evaluating oral contraceptive use as a risk factor for all cervical carcinomas failed to show a relationship [11]. In this study population, $47 \%$ of married women under 45 years old had used oral contraceptives. In 1973 the estimated use of oral contraceptives for married females in the U.S. population was $25 \%$ [12]. The difference between this study population and the national estimate is not statistically significant $(P=0.28)$. Abnormal bleeding in $71 \%$ remains the most common symptom of this disease with previous reports showing 74 and $88 \%$. The majority of women in this study had normal pelvic exams within 1 to 3 years of diagnosis and almost half had normal cytology. The triad of normal exam, normal cytology, and unexplained abnormal bleeding appears to be the hallmark of early cervical adenocarcinoma. In gynecologic follow-up an endocervical curettage may help detect this abnormality in patients with these findings.

Radical surgery, radiation therapy, or a combined approach offered equal survival in this scrics. The most significant factor influcncing prognosis appears to be lymph node status. The presence of metastatic disease to lymph nodes is a grave prognostic feature and this low survival is similar to that reported by Berek et al. [13]. This is in contradistinction to squamous cell metastases in lymph nodes where a 60\% 5-year survival in stage I disease has been reported [14]. Tumor grade significantly influences prognosis and these results are consistent with other reports where well-differentiated adenocarcinoma enjoyed the best survival [15].

When analyzed independently size appears to be a significant prognostic factor in early stage disease and the lowered survival in this series for lesions greater than $3 \mathrm{~cm}$ is consistent with previous reports $[8,16]$. In the multivariant analysis, however, the lesion size did not maintain its significance while nodal metastases overwhelmingly influenced survival. The presence of nodal metastases may suggest systemic disease and be the critical determinant in survival. Thus, the primary mode of therapy may need to include systemic therapy when this finding is encountered.

The preservation of ovarian function appears to be reasonable at the time of radical hysterectomy. The removal of ovaries did not influence survival and in those cases where salpingo-oophorectomy was performed no case of ovarian metastases was found. Gallup and Abell found metastatic disease to the ovary in one of seven patients undergoing radical hysterectomy with bilateral salpingooophorectomy [1]. The removal of ovaries in young women undergoing radical hysterectomy may not be mandatory, but when preserved careful inspection is necessary.

\section{SUMMARY}

Adenocarcinoma of the cervix appears to be increasing in incidence and represents a very real challenge for early diagnosis and improved treatment modalities. Early diagnosis is hindered by lack of clinical findings and normal cytologic 
screening. Survival is influenced by stage at presentation, grade, and presence of node metastases. Size by itself is a significant risk factor, but may be less important when controlled for lymph node status. Radical hysterectomy, radiation therapy, and combined surgery with radiation offered equal survival in stage I disease. Ovarian conservation did not adversely influence survival and should be considered in young women undergoing radical hysterectomy. Further investigation is necessary if survival rates similar to squamous cell disease are to be achieved especially in advanced stage disease and in patients with metastatic lymph node disease.

\section{REFERENCES}

1. Gallup, D. G., and Abell, M. R. Invasive adenocarcinoma of the uterine cervix, Obstet. Gynecol. 49, 596 (1977).

2. Davis, J. R., and Moon, L. B. Increased incidence of adenocarcinoma of uterine cervix, Obstet. Gynecol. 45, 79 (1975).

3. Kaplan, E. L., and Meier, P. Nonparametric estimation from incomplete observations, J. Amer. Stat. Assoc. 53, 457 (1958).

4. Breslow, N. A generalized Krushal Wallis test for comparing $\mathrm{K}$ samples subject to unequal patterns of censorship, Biometrikes 57, 579 (1970).

5. Cox, D. R. Regression models and life tables, J. R. Stat. Soc. Brit. 34, 187 (1972).

6. Abell, M. R., and Gosling, J. R. Gland cell carcinoma (adenocarcinoma) of the uterine cervix, Amer. J. Obstet. Gynecol. 83, 729 (1963).

7. Fu, Y. S., Reagan, J. W., Wentz, W. B., et al. Adenocarcinoma and mixed carcinoma of the uterine cervix, Cancer 49, 2560 (1982).

8. Shingleton, H. M., Gore, H., Bradley, P. H., et al. Adenocarcinoma of the cervix, Amer. J. Obstet. Gynecol. 139, 799 (1981).

9. Kjorstad, K. E. Adenocarcinoma of the uterine cervix, Gynecol. Oncol. 5, 219 (1977).

10. Current estimates from the national health interview, National Center for Health Statistics (1975).

11. Boyce, J. M., Lu, T., Nelson, J. Il., et al. Oral contraceptives and ccrvical carcinoma, Amer. J. Obstet. Gynecol. 128, 761 (1977).

12. Bachrach, C., and Mosher, W. Use of contraception in the United States 1982, Advanced Data No. 102, National Center for Health Statistics (Dec. 4, 1984).

13. Berek, J. S., Castaldo, T. W., Hacker, N. F., et al. Adenocarcinoma of the uterine cervix, Cancer 48, 2734 (1981).

14. Morley, G. W., and Seski, J. C. Radical pelvic surgery vs radiation therapy for stage I carcinoma of the cervix, Amer. J. Obstet. Gynecol. 126, 785 (1976).

15. Berek, J. S., Hacker, W. F., Fu, Y. S., et al. Adenocarcinoma of the uterine cervix: Histologic variables associated with lymph node metastases and survival, Obstet. Gynecol. 65, 46 (1985).

16. Rutledge, F. N., Galakatoc, A. B., Wharton, J. T., et al. Adenocarcinoma of the uterine cervix, Amer. J. Obstet. Gynecol. 122, 236 (1975). 